\title{
RESEARCH AND REPORTS
}

\section{Does Race Alter the Relationship Between HbAlc and Glucose in Type 2 Diabetes?}

\author{
KRISTINA JACKSON BEHAN, JUSTICE MBIZO, MICHAEL A. JOHNSTON, \\ MARCIA DUMAS, MARISA C. YATES
}

\begin{abstract}
Objective: Hemoglobin A1c (HbA1c) is the standard measurement of glycemic control, and the HbA1c value can be used to estimate average glucose using a formula. Several studies suggest that the relationship between average glucose and $\mathrm{HbA} 1 \mathrm{c}$ may be different for Blacks. This project enrolled non-Hispanic black and white individuals with type 2 diabetes and evaluated the relationship between $\mathrm{HbA1c}$ and blood glucose.

Method: 22 black and 29 white adults with type 2 diabetes were included in the analysis. Approximately 42 measurements (fasting and postprandial glucose) were collected over three months and compared to HbA1c of the third month. The effect of race was evaluated by ANCOVA and $\chi^{2}$ analysis testing the slope and intercepts simultaneously for $\mathrm{HbAlc}$ and its relationship to fasting glucose and to postprandial glucose.
\end{abstract}

Results: The relationship between HbA1c and glucose was not statistically significantly different between Blacks and Whites (ANCOVA: $P=0.968$ for fasting glucose, $P=0.428$ for postprandial glucose), allowing us to calculate estimated fasting and postprandial glucose disregarding race. For fasting glucose, the linear regression is $\mathrm{FG}_{\mathrm{mg} / \mathrm{dl}}=(18.939 \mathrm{X} \mathrm{HbA1c} \%)-1.864, \mathrm{R}^{2}$ $=0.586, P<0.0001$. For postprandial glucose, the linear regression is $\ln \left(\mathrm{PPG}_{\mathrm{mg} / \mathrm{dl}}\right)=(1.261 \mathrm{X}$ $\ln (\mathrm{HbA} 1 \mathrm{c} \%))+2.555, \quad \mathrm{R}^{2}=0.614, \quad P<0.0001$. Predicted values for postprandial glucose based on $\mathrm{HbA1c}$ were similar to estimated average glucose values reported by ADAG.

Conclusion: This study reinforces the A1c-Derived Average Glucose (ADAG) group finding that the relationship between $\mathrm{HbA1c}$ and glucose is similar in non-Hispanic black and white adults with type 2 diabetes.

ABBREVIATIONS: FG: fasting glucose; PPG: postprandial glucose; HbA1c: Hemoglobin A1c; eAG: estimated average glucose; ADAG: A1c Derived Average
Glucose Study; UKPDS: United Kingdom Prospective Diabetes Study; NHANES: National Health and Nutrition Examination Survey; SIGT: Screening for Impaired Glucose Tolerance Study; DCCT: Diabetes Control and Complications Trial; AUC: area under the curve; HPLC: high performance liquid chromatography

INDEX TERMS: Adult, African Continental Ancestry Group, African American, Black, Caucasian, Chi-square test, Diabetes Mellitus/blood, Diabetes Mellitus/ ethnology, Diabetes Mellitus, Type 2, European Continental Ancestry Group, Glycated hemoglobin, Glucose, Health disparities, Hemoglobin A, Glycosylated/metabolism, Hemoglobin A1c, Linear regression analysis, Longitudinal Studies, Minority health, Prospective Studies, Racial Bias, White

Clin Lab Sci 2014;27(2):89

Kristina Jackson Behan PhD, MLS(ASCP), University of West Florida, School of Allied Health and Life Sciences, Pensacola, FL

Justice Mbizo DrPH, University of West Florida, School of Allied Health and Life Sciences, Pensacola, FL

Michael A. Johnston, MS, University of West Florida, School of Allied Health and Life Sciences, Department of Math and Statistics, Pensacola, FL

Marcia Dumas MEd, MLS(ASCP), West Florida Hospital Laboratory, Pensacola, FL

Marisa C. Yates, MA, Miami Dade College, Institutional Research, Miami, FL

Address for Correspondence: Kristina Jackson Behan PhD, MLS(ASCP), University of West Florida, School of Allied Health and Life Sciences, Building 58, 11000 University Parkway, Pensacola, FL, 32514, (850) 474- 
3060,kbehan@uwf.edu

\section{INTRODUCTION}

In the United States, the prevalence of type 2 diabetes in non-Hispanic Black adults is approximately $18.7 \%$ compared to $10.2 \%$ in non-Hispanic whites. ${ }^{1}$ Blacks are more likely to suffer from severe complications of diabetes, such as amputations, blindness and end stage renal failure than their white counterparts. ${ }^{2}$ However, these complications can be ameliorated by tight glucose control. ${ }^{3-5}$ Hemoglobin A1c (HbA1c) has a linear relationship with blood glucose which permits an estimation of average glucose (eAG) to be calculated, such that eAG $(\mathrm{mg} / \mathrm{dl})=(28.7$ X HbA1c\% $)-46.7 .^{6,7}$ Current diabetes management guidelines recommend that HbAlc should be performed every 3-6 months ${ }^{8}$. The target goal for $\mathrm{HbAlc}$ for otherwise healthy individuals is $6.5-7.0 \%(48-53 \mathrm{mmol} / \mathrm{mol})$, and $<7.5$ - 8.0\% (58-64 $\mathrm{mmol} / \mathrm{mol})$ for older individuals who are more likely to have adverse events due to multiple medications or consequences of hypoglycemia.' Selfmonitoring of blood glucose is also recommended, but the frequency and timing of such for individuals with type 2 diabetes has not been agreed upon. ${ }^{8}$

It is not clear from previous studies whether HbAlc predicts the same average glucose in Blacks with type 2 diabetes as it does in Whites. Minorities are underrepresented in the classic studies that defined the relevance of HbA1c. The U.K. Prospective Diabetes Study (UKPDS), for example, showed that a reduction in microvascular complications of diabetes could be achieved by tight glucose control. However, only $9 \%$ of the subjects were classified as Afro Caribbean ethnicity. ${ }^{4}$ The A1c-Derived Average Glucose (ADAG) group tested 507 subjects using continuous interstitial glucose monitoring and $\mathrm{HbAlc}$ to establish the linear correlation of the two; only $4 \%$ of these subjects were African/ African Americans with type 2 diabetes. $^{7}$ The Diabetes Control and Complications Trial that demonstrated improved microvascular outcomes from tight glycemic control was limited to individuals with type 1 diabetes. ${ }^{3}$ The Action to Control Cardiovascular Risk in Diabetes (ACCORD) study reported that intensive glucose control delayed the onset of albuminuria, cataracts and neuropathy, but did not distinguish results based on the racial makeup of the study population. ${ }^{5}$ Data from the Third National Health and Nutrition Examination Survey (NHANES-
3) and the Screening for Impaired Glucose Tolerance Study (SIGT) showed that Blacks had a higher HbA1c than Whites in individuals without diabetes, those with impaired glucose and those with diabetes, even after adjustment for fasting plasma glucose levels. ${ }^{10}$ Similarly, Blacks with impaired glucose tolerance enrolled in the Diabetes Prevention Program had higher HbA1c values than Whites, in spite of lower glucose AUC values. ${ }^{11}$ Those authors questioned the validity of $\mathrm{HbAlc}$ as a diagnostic tool for diabetes in Blacks because of this discrepancy.

The current report used a community based longitudinal study design as a pilot study to determine if the relationship between blood glucose and HbA1c is the same in both non-Hispanic Blacks $(\mathrm{n}=22)$ and non-Hispanic Whites $(\mathrm{n}=29)$ with type 2 diabetes. Participants produced sets of fasting and 2 hour postprandial self-monitoring blood glucose values over a three month period, and these were correlated to HbA1c values. Since the presence of iron deficiency anemia and some hemoglobinopathies are known to interfere with $\mathrm{HbA1c}$ testing, data from individuals with those conditions was not included in the analysis.

\section{MATERIALS AND METHODS Study population}

The study used a community based population composed of non-Hispanic Black and non-Hispanic White men and women 48 years and older with a known diagnosis of diabetes mellitus. The study was approved by the Institutional Review Board at the University of West Florida. All participants signed an informed consent. The study was observational in nature, and diabetes management was left to the individuals under the direction of their own physicians. Black and White participants were recruited at churches, schools and community events in Pensacola, Florida from 2008-2011. All participants were ambulatory.

We attempted to balance the study with respect to race. Eighty five individuals enrolled in the study. All participants were evaluated for hemoglobinopathy and iron deficiency anemia by hemoglobin electrophoresis and complete blood count (CBC). Hemograms were collected four times over the course of the study to verify consistency. Reasons for exclusion from the final analysis included insufficient data (4) or the presence of 
Hemoglobin S, C or elevated F (7). Fifteen participants withdrew for health or undisclosed personal reasons. Participants with a HbAlc > 14\% (130 mmol $/ \mathrm{mol})$ were also excluded since this value exceeded the limitations of the analyzer. Two participants showed a significant change between $\mathrm{HbAlc}$ at baseline and the subsequent values. Those participants continued to be studied for one extra month, and their values from the first month were dropped. Participants who otherwise showed greater than $1.5 \%$ variability in $\mathrm{HbA} 1 \mathrm{c}$ from baseline to endpoint were excluded from the analysis. The final analysis was performed on the 51 individuals who remained.

\section{Questionnaire}

At the initial meeting, participants completed a questionnaire about demographics, time since diagnosis of diabetes, diagnosis of hypertension, therapy for diabetes, height and weight, and history of bleeding or anemia. Participants self-identified their race and gave their own assessment of general health.

\section{Laboratory Measurements}

Participants were provided with a Bayer Contour Glucose meter with Ascensia strips, (Bayer HealthCare, Daphne, AL), and instructed to collect glucose measurements two weeks out of each month. The Ascensia strip utilizes glucose dehydrogenase and coenzyme pyroloquinoline quinone to measure glucose in fingerstick whole blood. Imprecision of the meters was determined to be $1.2 \%$ at $105 \mathrm{mg} / \mathrm{dL}(5.83$ $\mathrm{mmol} / \mathrm{l}), 3.1 \%$ at $224 \mathrm{mg} / \mathrm{dL}(12.44 \mathrm{mmol} / \mathrm{l})$ and $5.9 \%$ at $332 \mathrm{mg} / \mathrm{dL}(18.44 \mathrm{mmol} / \mathrm{l})$. The meter applies an automatic correction to report results as plasma glucose.

In our study, subjects collected fasting morning glucose for one week, followed by one week of two hour postprandial (lunch or dinner) glucose measurements for three months. Subjects were encouraged to continue to test as their physician recommended during the two non-specified weeks of each month. Participants were contacted by phone and/or email with reminders over the course of the study. An average of the fasting glucose (FG) for the 3 months was used in the regression for $\mathrm{FG}$; an average of the postprandial glucose (PPG) for the 3 months was used in the regression for the PPG.

HbA1c was tested at baseline, after two months, three months and four months. HbA1c values from the third month were used in the correlation. HbAlc was performed by immunoassay on a Siemens (formerly Bayer, Tarrytown, NY) DCA 2000. The DCA is certified by the National Glycohemoglobin Standardization Program as a method traceable to the Diabetes Control and Complications Trial reference method. ${ }^{12}$

Imprecision of the analyzer was determined to be $4.3 \%$ at $5.7 \% \mathrm{HbA} 1 \mathrm{c}(39 \mathrm{mmol} / \mathrm{mol})$ and $2.5 \%$ at $11.7 \%$ HbAlc $(104 \mathrm{mmol} / \mathrm{mol})$. Participants were tested for hemoglobinopathy by electrophoresis using Paragon Hemoglobin electrophoresis (Beckman Coulter, Fullerton, CA). Hemograms were performed using an AcT analyzer (Beckman Coulter).

\section{Statistical Analysis}

We used SAS Version 9.2 (Cary, NC) for the analysis and Excel 2010 (Microsoft, Seattle WA) to generate the regression lines. We utilized both the $\chi^{2}$ test comparing the intercept and slope of the regression lines simultaneously and Analysis of Covariance (ANCOVA). The $\chi^{2}$ test directly replicates the analysis performed in the ADAG study ${ }^{7}$ and provided additional concordance for the ANCOVA results. The ANCOVA was chosen as it represents a robust method of analyzing regression differences between groups, correcting for additional variability that may be introduced by extraneous variables (covariates). Its particular strength is that it gives a result that is unbiased regardless of the baseline distribution of the prognostic variables. ${ }^{13}$ ANCOVA also provides increased power of the trial and precision of the treatment effect. Since HbA1c levels are known to proportionally increase with blood glucose levels they were included as a covariate in the ANCOVA. Additionally, postprandial data was lntransformed to normalize the data, including residuals from the analysis.

Linear, quadratic, and exponential regression models were applied to estimate the relationship between $\mathrm{HbA1c}$ levels in both fasting and postprandial glucose levels independently. There was no major statistical difference between the linear and quadratic models. The exponential model produced too much variability when estimating blood glucose in the higher and lower ranges of HbA1c. We therefore compared linear regression lines of Blacks and Whites in fasting and postprandial 
blood glucose data. Before testing for the effects of HbA1c on blood glucose in the two different populations, homogeneity of regression slopes between treatments was confirmed. (For Blacks vs Whites: Fasting Blood Glucose: $F_{1,47}=0.642, P=0.427$; for Blacks vs Whites: Postprandial Blood Glucose: $F_{1,47}=$ $0.288, P=0.594)$.

\section{Role of the Funding Source}

The funding sources had no role in the study design, conduct, or interpretation of results.

\section{RESULTS}

Fifty one participants completed the study. Demographics are presented in Table 1. The mean age among the Blacks was 64.3 years (SD: 10.8) compared to 59.2 (SD: 8.4) among the Whites. More black participants had a diagnosis of hypertension. More white participants were treated with oral medication than black participants. More white participants considered themselves in good health. RBC and hemoglobin was higher in white participants than in black participants. Fasting and postprandial glucose measurements were similar for both groups.

The relationship between $\mathrm{HbA1c}$ and fasting blood glucose $\left(A N C O V A: F_{1,48}=0.002, P=0.968\right)$ showed no statistically significant difference between Blacks and Whites at a P-value of 0.05 . The $\chi^{2}$ test with 2 degrees of freedom comparing the intercepts and slopes on each line simultaneously for fasting blood glucose was also non-significant $\left(\chi^{2}=0.32, P=0.7265\right)$. The relationship between $\mathrm{HbA1c}$ and postprandial blood glucose also showed no statistically significant difference between Blacks and Whites at the same P-value in both the ANCOVA (ANCOVA : $F_{1,48}=0.640, P=0.428$ ) and the $\chi^{2}$ test $\left(\chi^{2}=1.00, P=0.3745\right)$.

The relationship between HbAlc levels and Fasting Glucose, expressed in terms of a simple linear regression independently for Blacks is Fasting Blood Glucose (B) mg/dl $=(21.707 \mathrm{X} \mathrm{HbA1c} \%)-20.850, \mathrm{R}^{2}=0.5889, P<$ 0.0001 . The simple linear regression for Whites is Fasting Blood Glucose $(W)_{\text {mg/dl }}=(17.705$ X HbA1c\% $)+$ $6.726, \mathrm{R}^{2}=0.5925, P<0.0001$.

The relationship between the HbA1c levels and the Fasting Glucose (FG) measurements was statistically indistinguishable between the two groups; this allowed us to calculate an estimated fasting glucose for HbA1c levels independent of race. The relationship between HbA1c levels and fasting glucose for all subjects $(\mathrm{n}=51)$, expressed as the simple linear regression $F G_{m g / d l}=$ $\left(18.939\right.$ X HbA1c\%) - 1.864, $\mathrm{R}^{2}=0.586, P<0.0001$ can be seen in Figure 1.

Table 1. Population characteristics

\begin{tabular}{lcc}
\hline & Non-Hispanic & Non-Hispanic \\
& White & Black \\
Sample size (n) & 29 & 22 \\
Age in years & $59.2(8.4)$ & $64.3(10.8)$ \\
BMI (kg/m2) & $31.4(5.2)$ & $31.7(6.4)$ \\
Diagnosis of & $72.4 \%$ & $81.8 \%$ \\
hypertension \% & & \\
Take medication for & $70.0 \%$ & $81.8 \%$ \\
hypertension \% & & \\
Female \% & $51.7 \%$ & $77.3 \%$ \\
RBC (X 10 $12 / \mathrm{L})$ & $4.69(0.49)$ & $4.49(0.45)$ \\
Hemoglobin (g/dl) & $14.0(1.5)$ & $12.7(1.1)$ \\
MCV (fl) & $90.4(4.1)$ & $89.3(6.1)$ \\
Consider self to be & $96.6 \%$ & $72.7 \%$ \\
in good health & & \\
Treatment for Diabetes & & $68.2 \%$ \\
Oral medication \% & $75.9 \%$ & $22.7 \%$ \\
Insulin \% & $20.7 \%$ & \\
Years since diagnosis of & $8.5(6.9)$ & \\
diabetes & & \\
HbA1c \% & $7.0(1.4)$ & $6.9(6.4)$ \\
HbA1c mmol/mol & $53.41(15.2)$ & $52.04(11.7)$ \\
Fasting glucose (mg/dl) & $131.4(32.2)$ & $129.1(30.6)$ \\
Fasting glucose (mmol/l) & $7.30(1.79)$ & $7.17(1.70)$ \\
Postprandial glucose (mg/dl) & $150.2(40.4)$ & $154.3(51.7)$ \\
Postprandial glucose (mmol/l) & $8.34(2.25)$ & $8.57)$ \\
& & \\
\hline
\end{tabular}

Data are mean $(\mathrm{SD})$ unless otherwise indicated

The relationship between HbAlc levels and Postprandial Glucose, expressed in terms of a simple linear regression independently for Blacks is $\ln$ (Postprandial Blood Glucose $)(B)_{\text {moldl }}=(0.142 \mathrm{X}$ $\ln (\mathrm{HbA} 1 \mathrm{c} \%))+3.980, \mathrm{R}^{2}=0.619, P<0.0001$. The simple linear regression for Whites is $\ln$ (Postprandial Blood Glucose $\left.(W)_{\text {mgld }}\right)=(1.210 \times \ln (\mathrm{HbA} 1 \mathrm{c} \%))+$ 2.638, $\mathrm{R}^{2}=0.709, P<0.0001$.

The relationship between the HbAlc levels and the Postprandial Glucose (PPG) measurements was statistically indistinguishable between groups; this allowed us to calculate an estimated postprandial glucose for HbA1c levels independent of race. The relationship between $\mathrm{HbAlc}$ levels and postprandial blood glucose for all subjects $(n=51)$, expressed as the 


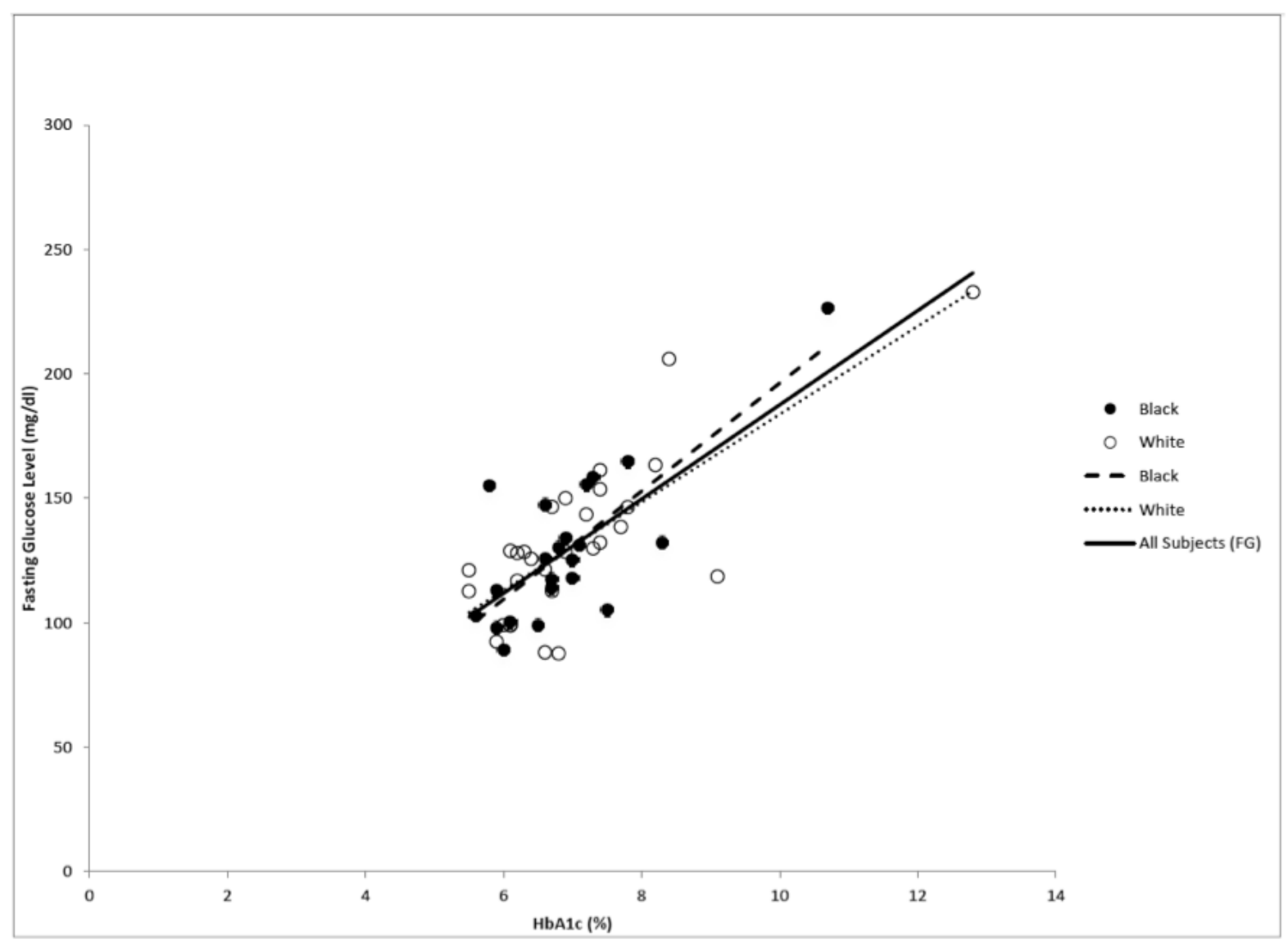

Figure 1. HbA1c versus Fasting Glucose. Relationship between HbA1c and Fasting Glucose for Blacks (--) and Whites (.....). Bivariate scatter plot of fasting glucose measurements in Blacks (black circles) and Whites (white circles). All points ( $\mathrm{n}=51$ ) were regressed to determine an equation to predict fasting glucose levels; Fasting Glucose $\mathrm{mg} / \mathrm{dl}=(18.939 \mathrm{X} \mathrm{HbA1c} \%)-1.864, \mathrm{R}^{2}=0.586, P<$ 0.0001 .

simple linear regression $\ln \left(P P G_{m g r d l}\right)=(1.261 \mathrm{X}$ $\ln (\mathrm{HbA} 1 \mathrm{c}))+2.555, \mathrm{R}^{2}=0.614, P<0.0001$ can be seen in Figure 2.

\section{DISCUSSION}

Data from the SIGT and NHANES suggested that HbA1c might not adequately reflect average glucose in Blacks. ${ }^{10,11}$ The small group representing Blacks with type 2 diabetes that was included in the ADAG study showed no statistically significant difference in the relationship compared to the relationship in Whites $\left(\chi^{2}\right.$ analysis, $P=0.07$ ). Due to the low enrollment and the small $P$ value, those authors recommended further study. ${ }^{7}$ The ADAG study group was comprised of 21 participants consisting of Africans from Cameroon and African-Americans. In our study, we enrolled 22 nonHispanic Blacks and 29 non-Hispanic Whites. We also found no difference between the Blacks and Whites with respect to the relationship between $\mathrm{HbA1c}$ and fasting or postprandial glucose. Both studies suffer from low enrollment, and these results bear repeating in a larger study.

We compared the translation of $\mathrm{HbA1c}$ to fasting glucose and separately to postprandial glucose. The postprandial glucose prediction was superior to fasting glucose; postprandial glucose closely approximated that determined by the ADAG group as estimated average glucose, with the difference in glucose approximately ranging between $1-3 \%$, shown in Table 2 . The finding that postprandial glucose is a good indicator of average glucose is in agreement with data from the DCCT, which showed that post-lunch glucose and later time points had a higher correlation to HbAlc than any premeal glucose measurements. ${ }^{6}$ 


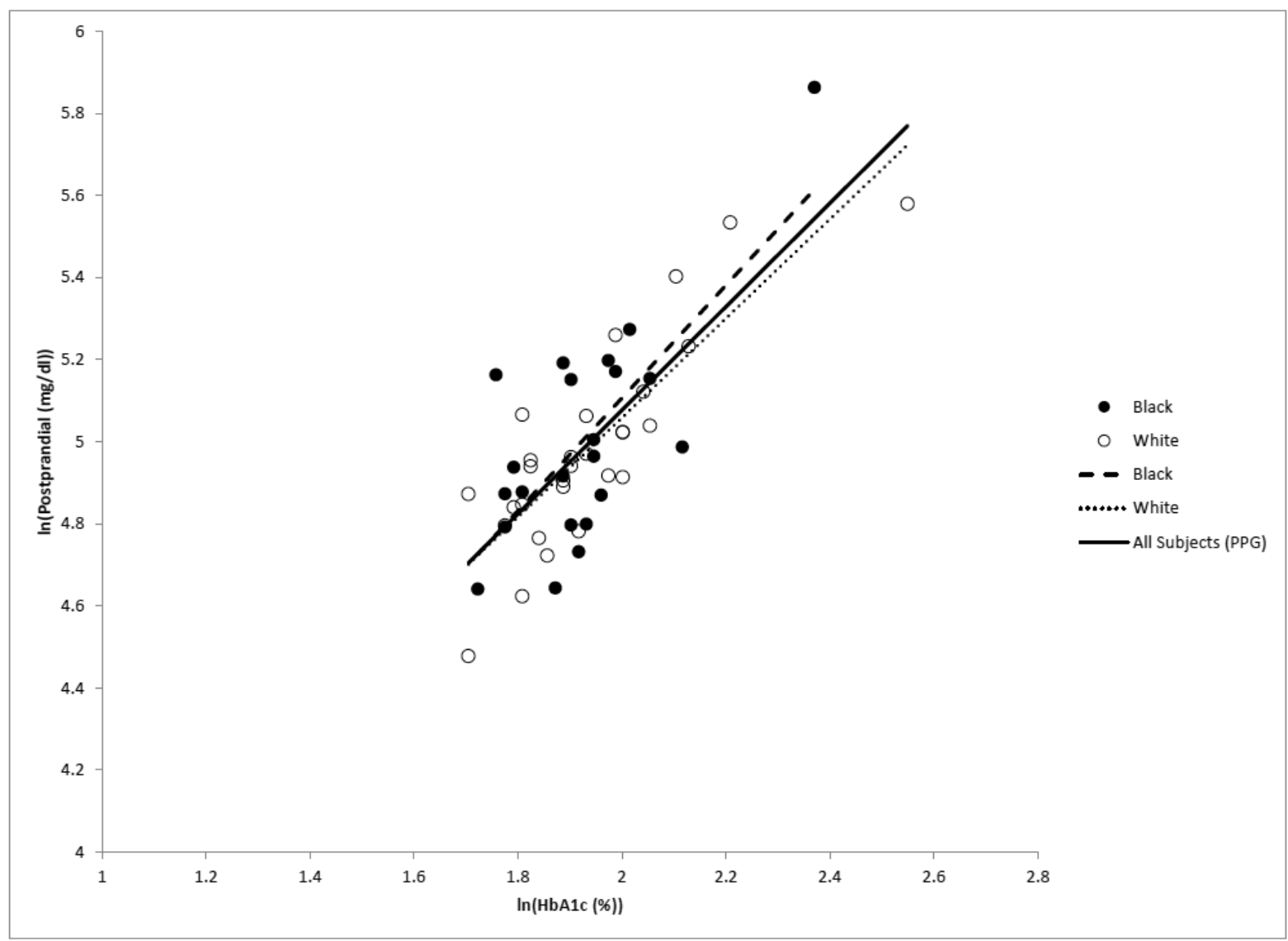

Figure 2. $\ln (\mathrm{HbA1c})$ versus $\ln$ (Postprandial Glucose). Relationship between $\ln (\mathrm{HbA1c})$ and $\ln$ (Postprandial Glucose) for Blacks (--) and Whites (.....). Bivariate scatter plot of fasting glucose measurements in Blacks (black circles) and Whites (white circles). All points $(\mathrm{n}=51)$ were regressed to determine an equation to predict postprandial glucose levels; $\ln ($ Postprandial Glucose $\mathrm{mg} / \mathrm{dl})=(1.261 \mathrm{X}$ $\ln (\mathrm{HbA} 1 \mathrm{c} \%))+2.555, \mathrm{R}^{2}=0.6142, \mathrm{P}<0.0001$.

Table 2. Estimated Postprandial Glucose compares with Estimated Average Glucose

\begin{tabular}{lccc}
\hline & $\begin{array}{c}\text { Postprandial } \\
\text { Regression* } \\
\text { A1C (\%) }\end{array}$ & $\begin{array}{c}\text { Estimated Average } \\
\text { Glucose** } \\
(\mathbf{m g} / \mathbf{d l})\end{array}$ & \% Difference \\
\hline 5 & $98(70-137)$ & $97(76-120)$ & $1.0 \%$ \\
6 & $123(89-170)$ & $126(100-152)$ & $2.4 \%$ \\
7 & $150(109-206)$ & $154(123-185)$ & $2.6 \%$ \\
8 & $177(128-245)$ & $183(147-217)$ & $3.3 \%$ \\
9 & $206(148-286)$ & $212(170-249)$ & $2.8 \%$ \\
10 & $235(167-329)$ & $240(193-282)$ & $2.1 \%$ \\
11 & $265(187-375)$ & $269(217-314)$ & $1.5 \%$ \\
12 & $295(206-423)$ & $298(240-347)$ & $1.0 \%$ \\
\hline
\end{tabular}

Data in parenthesis are $95 \%$ Confidence Intervals of the predicted value. * Linear regression of estimated average postprandial glucose calculated from $\ln ($ Postprandial Glucose $\mathrm{mg} / \mathrm{dl})=(1.261 \mathrm{X} \ln (\mathrm{HbA} 1 \mathrm{c}))+2.555$.

**Table 2 Estimated average glucose, Nathan et al. (7). To convert glucose to $\mathrm{mmol} / \mathrm{l}$, divide $\mathrm{mg} / \mathrm{dl}$ by 18 .
Strengths of this study are that the participants were enrolled for four months with the collection of 3 months of glucose measurements, the establishment that the subjects had a steady state of $\mathrm{HbAlc}$, and that the possible confounding effect of anemia, blood loss and hemoglobinopathy was eliminated. Participants used the same type of glucose meter, which corrects the value to estimate plasma glucose. Blood was not stored or transported, thereby preventing the effect of glycolysis. Our results are further strengthened by the finding that postprandial glucose predictions using our regression $\ln ($ Postprandial Glucose $\mathrm{mg} / \mathrm{dl})=(1.261 \mathrm{X}$ $\ln (\mathrm{HbA} 1 \mathrm{c} \%))+2.555$ compares closely with estimated average glucose using eAG $(\mathrm{mg} / \mathrm{dl})=(28.7$ X HbA1c\%) - 46.7 from the ADAG group. ${ }^{7}$ The major weakness of the study is the small number of participants. However, this is not uncommon in studies of this nature. In the comparison ADAG study, of 507 subjects, only 21 were Blacks with type 2 diabetes. Therefore, our results are 
an important addition to the findings of the ADAG study. Our results cannot be assumed to be correct for individuals with hemoglobinopathies or anemia, or to other races. Most of the individuals in the study had HbA1c values less than $10 \%$, therefore the conclusions may not be generalizable to individuals with higher HbA1c. This study enrolled adults with type 2 diabetes, and results may not be applicable to children or individuals with type 1 diabetes. Co-morbidities were not reported or analyzed, however most of the participants reported being in good health and all were in a community setting and ambulatory.

The recommended frequency of testing HbAlc is twice a year for patients in good control, and more frequently for those with poor control. ${ }^{8}$ Goals for fasting glucose are $70-130 \mathrm{mg} / \mathrm{dl}(3.89-7.22 \mathrm{mmol} / \mathrm{l})$ and postprandial glucose $<180 \mathrm{mg} / \mathrm{dl}(10.0 \mathrm{mmol} / \mathrm{l})$, but the need for frequent self-monitoring of blood glucose for patients on non-insulin therapy is controversial. Although more frequent testing can lead to improved glycemic control, some investigators believe that the improvement may be attributed to better education and self-care and not directly to the testing itself. ${ }^{8}$ Selftesting is expensive, and its cost was a common concern to the individuals in the study. The cost of glucose strips increased over the course of this study, and insurance reimbursement decreased, making frequent testing a financial concern for many patients. In our study, participants reported that it was easier for them to regularly perform fasting measurements than postprandial measurements, however, the postprandial results were better predictors of HbAlc. These limitations to self-testing and the ambiguous standards for frequency put more significance on regular, accurate $\mathrm{HbA1c}$ results for good medical care.

HbA1c measurement is approved for the diagnosis of diabetes and our results support its continued use in non-Hispanic Blacks as well as non-Hispanic Whites with this caveat: The Black population is more likely to have a hemoglobin variant or elevated Hemoglobin $\mathrm{F}$ that may lead to false interpretation of $\mathrm{HbAlc}$ values. HbA1c methods that utilize HPLC can identify those individuals, however immunoassays cannot. In this study, only individuals with normal hemoglobin electrophoresis patterns and normal red blood cell parameters were included. HbAlc screening for the diagnosis of diabetes in Blacks should be done with caution and with an understanding of the limitations of the methodology. ${ }^{14}$

ACKNOWLEDGEMENTS: Authorship Contribution: KJB designed the research and wrote the manuscript, and is the guarantor. MD performed laboratory analysis and reviewed the manuscript. JM recruited participants, performed statistical analysis, contributed to the discussion and reviewed/edited the manuscript. MAJ and MCY performed statistical analysis, and contributed to the discussion.

This project was supported by a grant from the American Society for Clinical Laboratory Sciences and the Scholarly and Creative Activities Board at the University of West Florida. Meters were kindly provided by Bayer Diabetes Care and the American Diabetes Association. Blood collection materials were provided by $\mathrm{BD}$. The authors have no conflicts of interest to disclose.

The authors thank the following for their contributions in statistical analysis: Arup K. Sinha, University of Texas, Anthony Okafor and Raid Amin, University of West Florida, and Alexander Bohnert, FriedrichAlexander-University of Erlangen-Nürnberg. For assistance with laboratory analysis and patient retention: Candice Rhodes, Brianna Goley, Christina Nelson, Hien Nguyen, Ruth Vargas, Mark De Luna, Tyrone Del Valle, Kelly Castagna and Manolya Rowe, University of West Florida. The authors thank the following for materials and discussion: Sherman Bonomelli, Ranga Rao, and Swarna Krothapalli, University of West Florida, Lynne Cranford from the American Diabetes Association, Maggie Sheldon from Bayer Diagnostics, Chris Tant from BD, and Trefor Higgins from DynaLifeDx.

\section{REFERENCES}

1. Centers for Disease Control and Prevention. 2011 National Diabetes Fact Sheet.

2. African Americans and Complications. American Diabetes Association. Accessed October 27, 2012. At www.diabetes.org/ living-with-diabetes/complicatons/african-americans-andcomplications.

3. The Diabetes Control and Complications Trial Research Group: The effect of intensive treatment of diabetes on the development and progression of long term complications in the diabetes control in insulin dependent diabetes mellitus. N Engl J Med. 1993;329;977-86.

4. UK Prospective Diabetes Study Group. Intensive blood- 
glucose control with sulphonylureas or insulin compared with conventional treatment and risk of complications in patients with type 2 diabetes (UKPDS 33). Lancet. 1998;352:837-53.

5. Ismail-Beigi F, Craven T, Banerji MA, Basile J, et al. Effect of intensive treatment of hyperglycaemia on microvascular outcomes in type 2 diabetes: an analysis of the ACCORD randomized trial. Lancet. 2010;376:419-30.

6. Rohlfing CL, Wiedmeyer H-M, Little RR, England JD, et al. Defining the Relationship Between Plasma Glucose and HbA1c. Diabetes Care. 2002;25:275-8.

7. Nathan DM, Kuenen J, Borg R, Zheng H, et al. Translating the A1C Assay into Estimated Average Glucose Values. Diabetes Care. 2008;31:1473-8.

8. American Diabetes Association. Standards of Medical Care in Diabetes - 2012. Diabetes Care. 2012;35: Supplement 1:S11S63.

9. Inzucchi SE, Bergenstal RM, Buse JB, Diamant $M$, et al. Management of Hyperglycemia in Type 2 Diabetes: A PatientCentered Approach. Position Statement of the American Diabetes Association (ADA) and the European Association for the Study of Diabetes (EASD). Diabetes Care. 2012;35:136479.

10. Ziemer DC, Kolm P, Weintraub WS, Vaccarino V, et al. Glucose-Independent, Black-White Differences in Hemoglobin A1c Levels, A Cross-sectional Analysis of 2 Studies. Ann Intern Med. 2010;152:770-77.

11. Herman WH, Ma Y, Uwaifo G, Haffner $S$, et al. Differences in A1c by Race and Ethnicity Among Patients With Impaired Glucose Tolerance in the Diabetes Prevention Program. Diabetes Care. 2007;30:2453-7.

12. National Glycohemoglobin Standardization Program. List of NGSP certified methods. Available at http://www.ngsp.org/ docs/methods.pdf. Accessed May 27, 2013.

13. Redmond CK, Colton T, eds. Biostatistics in Clinical Trials. Chichester, England: John Wiley \& Sons; 2001.

14. Behan KJ, Storey NM, Lee H-K. Reporting variant hemoglobins discovered during hemoglobin Alc analysis Common practices in clinical laboratories. Clin Chim Acta. 2009;406:124-8.

The peer-reviewed Research and Reports Section seeks to publish reports of original research related to the clinical laboratory or one or more subspecialties, as well as information on important clinical laboratory-related topics such as technological, clinical, and experimental advances and innovations. Literature reviews are also included. Direct all inquiries to Maribeth L. Flaws, Ph.D., SM(ASCP)SI, Associate Chairman and Associate Professor, Department of Medical Laboratory Science, Rush University Medical Center, 600 S Paulina Suite 1018A, Chicago IL 60612, Maribeth_L_Flaws@rush.edu. Clinical Laboratory Science encourages readers to respond with thoughts, questions, or comments regarding these articles. Email responses to westminsterpublishers@comcast.net. In the subject line, please type the journal issue and lead author such as "CLIN LAB SCI 27(2) RE BEHAN". Selected responses may appear in the Dialogue and Discussion section in a future issue. Responses may be edited for length and clarity. We look forward to hearing from you. 